\title{
Molecular Dynamics Simulation of Octacosane for Phase Diagrams and Properties via United Atom Scheme
}

\author{
Ling Dai ${ }^{1}$, Pavlo Rutkevych ${ }^{2}$, Souvik Chakraborty ${ }^{1}$, Gang $\mathrm{Wu}^{1}$, Jun $\mathrm{Ye}^{1}$, Y.H. Lau ${ }^{1}$, \\ Hariharaputran Ramanarayan ${ }^{1}$, and D.T. $\mathrm{Wu}^{1}$ \\ ${ }^{1}$ Institute of High Performance Computing \\ ${ }^{2}$ Institute of Chemical and Engineering Sciences
}

May 7, 2021

\begin{abstract}
We used united atom scheme to build three types of crystalline structures for octacosane (C28H58) and carried out molecular dynamic simulations to investigate their properties. By gradual heating the three polymorphs, we successfully reproduced the sequence of experimentally reported crystalline phase, intermediate rotator phase and liquid phase. The obtained structural properties of the phases, such as molecule chain morphology, density, chain tilt angle, cell anisotropy. We revealed three mechanisms which well described the kinetic deformation and expansion during the annealing process. Furthermore, our model successfully predicted the melting temperature and the heat of fusion. We also reproduced characteristics of the rotator phases and the liquid phase, indicating the transferability of the united atom scheme among different condensed phases of octacosane. Our methodology represents an effective and efficient means of numerical study for octacosane and may have implication for other members of the n-alkane family.
\end{abstract}

\section{Hosted file}

Manuscript_UA.pdf available at https://authorea.com/users/412365/articles/521079-moleculardynamics-simulation-of-octacosane-for-phase-diagrams-and-properties-via-united-atomscheme 